\title{
A Search for Brown Dwarfs around Young Solar-Analog Stars Using the Hōkūpa'a/Gemini Adaptive Optics System
}

\author{
Daniel E. Potter, Eduardo L. Martín, and Michael C. Cushing \\ Institute for Astronomy, University of Hawai' $i$, 2680 Woodlawn Drive, \\ Honolulu, HI, 96822
}

\begin{abstract}
We present the results of a search for low-mass companions around a sample of young, solar-analog stars using the Hōkūpa'a adaptive optics instrument mounted on the Gemini North $8 \mathrm{~m}$ telescope. Out of 31 stars observed, one binary brown dwarf system was found as a companion to the star HD 130948 (HIP 72567), as confirmed by proper motion and near-IR spectra. Orbital motion between the two brown dwarfs was measured, but our 14 month time baseline is inadequate to accurately measure the system's dynamical mass. Upcoming spectroscopic observations of the brown dwarfs will measure their lithium absorption lines to provide a more accurate age estimate of the system. The eventual dynamical mass determination coupled with the age determination will provide a valuable check of brown dwarf evolutionary models.
\end{abstract}

\section{Introduction}

A survey of 31 young $(<1 \mathrm{Gyr})$, nearby $(\sim 25 \mathrm{pc})$ solar analog stars, primarily selected from the catalog of Gaidos (2000), was carried out using the Hōkūpa'a adaptive optics system mounted on the Gemini North telescope. The instrument was operated in the dual imaging polarimetry mode (Potter 2002) because the primary goal of the survey was to place limits on the scattered light from potential debris disks around these stars. The only significant effect the polarimetry mode had on the brown dwarf survey was the reduction of the field-of-view to $\sim 3^{\prime \prime}$. 0 .

Of the 31 stars observed in the sample, 2 were found to have companions (HD 72760 and HD 130948). The companion around HD 72760 is located at a distance of 1 ." 0 from the primary but is too bright to be a substellar object (see Figure 1). HD130948 was found to have two companions (Figure 2) that are very closely separated from each other $(135 \mathrm{mas})$, with follow up proper motion observations and spectroscopy confirming they are true brown dwarf companions (Potter et al. 2002; Goto et al. 2002). Because the two brown dwarfs are likely gravitationally bound to each other with an estimated orbital period of about 10-20 years, the dynamical masses can be obtained through well sampled astrometric observations with sufficient time baseline and from the known distance from Hipparcos observations $(\mathrm{D}=17.9 \mathrm{pc})$. 


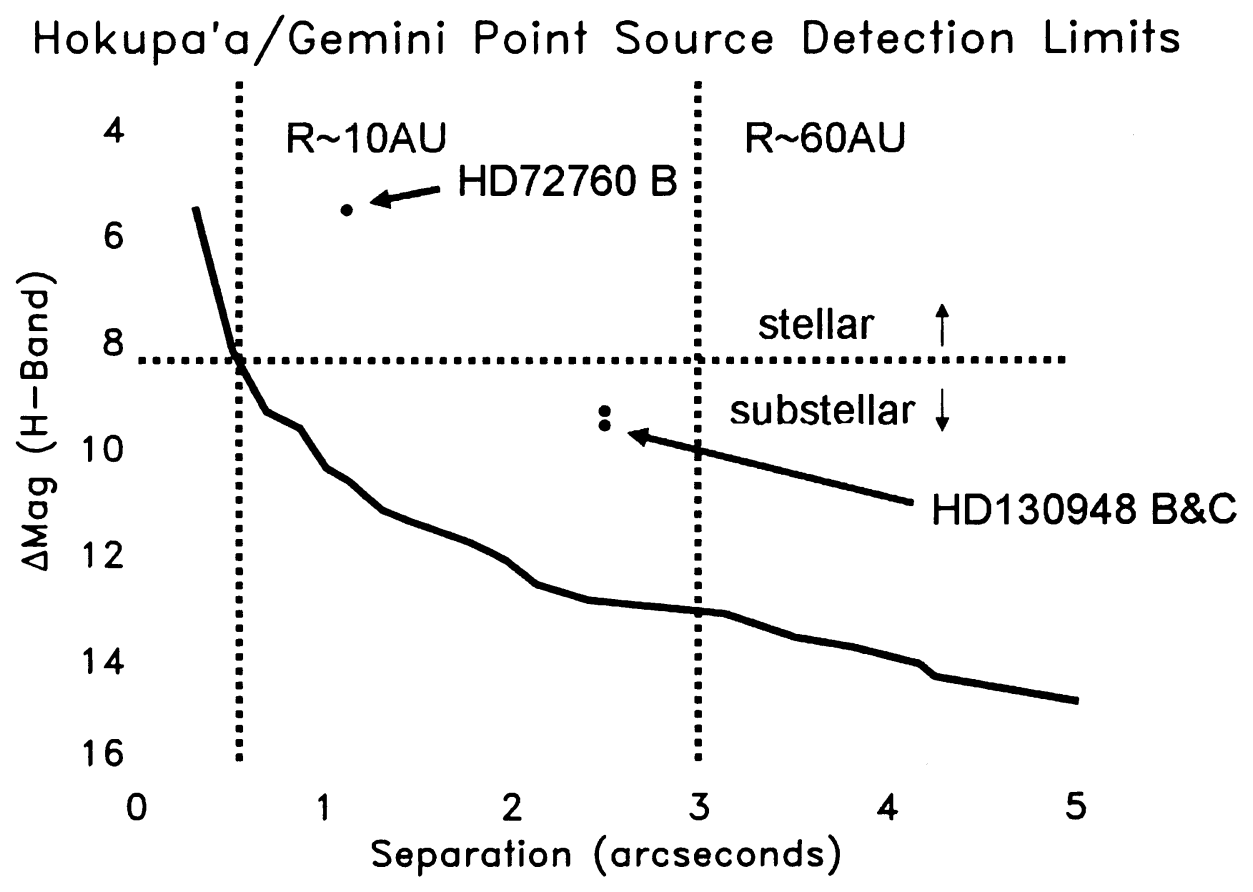

Figure 1. Point source detection limits versus radius from the PSF center. The horizontal dotted line indicates the approximate contrast required to detect a substellar mass object around a $0.5 \mathrm{Gyr}$ old solartype star based on Burrows et al. (2002). The vertical dotted lines mark the radial range where the survey is sensitive to detecting the brightest brown dwarfs (a polarimetry field stop cuts the field off at $33^{\prime \prime} 0$ ). This equates to radii between 10-60 AU with the stars at a distance of $\sim 25 \mathrm{pc}$. The low-mass companions found in the survey around HD 72760 and HD 130948 are labeled with arrows.

\section{Survey Sensitivity}

The Hōkūpa'a AO system (Graves et al. 2000) consistently delivers near diffraction limited images (Strehl $~ 0.2$ ) in the H-Band on the Gemini North 8 meter Telescope. The point source detection limit as a function of radius from the central star was obtained by inserting fainter versions of the AO PSF at random position angles, but at fixed radii, from the central star and finding the faintest artificial companion which could be consistently recovered by eye (Figure 1).

\section{Survey Results}

HD130948 B-C is the first brown dwarf binary system imaged around a G-type star. The detection of this new binary brown dwarf system in our survey gives a $3.2 \% \pm 3.2 \%$ frequency of brown dwarfs in our sensitivity region from Figure 


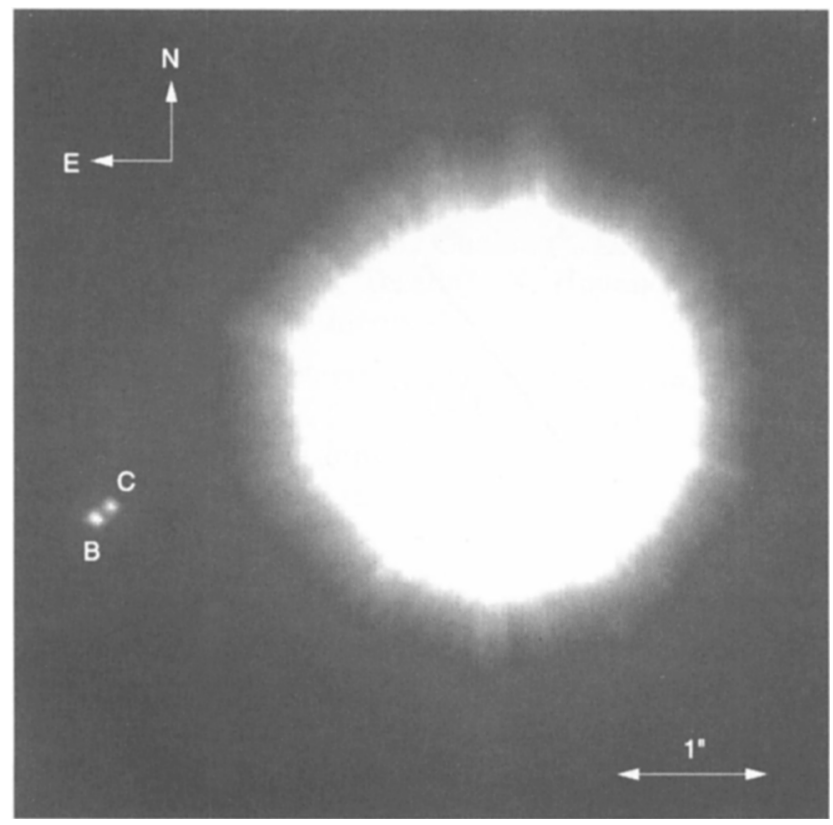

Figure 2. The discovery image (Potter et al. 2002) clearly resolves the companion to HD 130948 as a binary with a 135 mas separation. The companion is $\sim 8$ magnitudes fainter than the primary star in the $\mathrm{H}$ band at a separation of 2 .'64.

1. This number is likely a lower limit as we are not sensitive to lower mass, fainter, brown dwarfs. Gizis et al. (2001) reported a frequency of brown dwarf companions to G-type stars of $18 \% \pm 14 \%$ for separations larger than $1000 \mathrm{AU}$. Liu et al. (2002) have found an L-type companion at 14 AU from a G-type star using adaptive optics. Considering our result in conjunction with that of Gizis et al. and Liu et al., we suggest that brown dwarf companions to G-type dwarfs with separations larger than $10 \mathrm{AU}$ may be common, acknowledging that the survey is still in an uncertain regime limited by small number statistics.

In hopes of detecting orbital motion, we observed the binary companion over a 14 month baseline. We detected a relative position change of 25 mas (Figure 3) and interpret this change as orbital motion. Although this is insufficient to make dynamical mass measurements, and astrometric observations must be carried out in forthcoming years to construct the orbit and obtain a dynamical mass, it does offer promise that a dynamical mass will be obtained eventually. The binary brown dwarf companion to HD130948A will aid in the mass calibration of evolutionary models of brown dwarfs. Furthermore, the system will test the age dependence of the models through upcoming lithium absorption measurements scheduled to be obtained with HST/STIS.

Support for this work was provided by the National Aeronautics and Space Administration (NASA) grant NAG5-9992 and National Science Foundation (NSF) grant AST-0205862. 


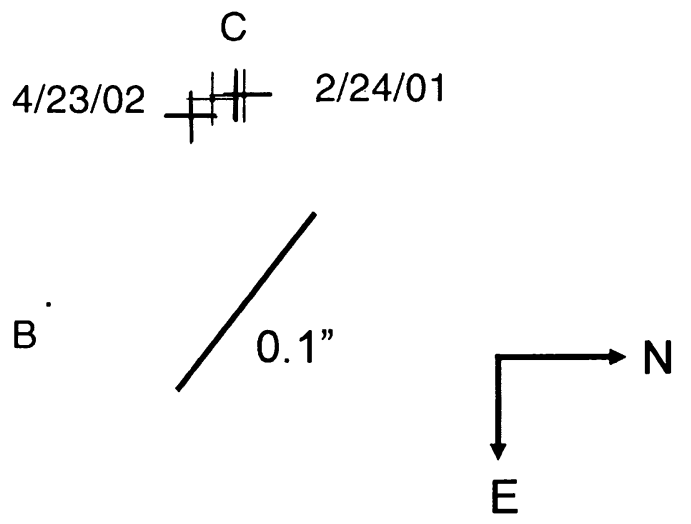

Figure 3. The relative astrometry between the two brown dwarf companions to HD 130948A plotted over a 14 month baseline shows a movement of $\sim 25$ mas. This motion is a confirmation that the two brown dwarfs are gravitationally bound, but at present, there is not enough orbital information known to make an accurate mass estimate. Follow-up observations are needed to accurately measure the mass of the brown dwarfs.

\section{References}

Burrows, A., Ram, R. S., Bernath, P., Sharp, C. M., \& Milsom, J. A. 2002, astro-ph 0206159

Gaidos, E. J., Henry, G. W., \& Henry, S. M. 2000, AJ, 120, 1006

Gizis, J. E. et al. 2001, ApJ, 551, 163

Goto, M. et al. 2002, ApJ, 567, 59

Graves, J. E. et al. 2000, SPIE, 4007, 26

Kirkpatrick, J. D. et al. 1999, ApJ, 519, 802

Liu, M. C. et al. 2002, ApJ, 571, 519

Potter, D. E. et al. 2002, ApJ, 567, L133 